Pacific

Journal of

Mathematics

\title{
A LOWER BOUND FOR EIGENVALUES OF THE POLY-LAPLACIAN WITH ARBITRARY ORDER
}

QING-Ming Cheng, Xuerong QI AND Guoxin WeI 


\title{
A LOWER BOUND FOR EIGENVALUES OF THE POLY-LAPLACIAN WITH ARBITRARY ORDER
}

\author{
Qing-Ming Cheng, Xuerong Qi And Guoxin Wei
}

\begin{abstract}
We study eigenvalues of the poly-Laplacian of arbitrary order on a bounded domain in an $\boldsymbol{n}$-dimensional Euclidean space. We obtain a lower bound for these eigenvalues, significantly improving on that of Levine and Protter. In particular, the result of Melas (2003) is subsumed.
\end{abstract}

\section{Introduction}

Let $\Omega \subset \mathbb{R}^{n}$ be a bounded domain with piecewise smooth boundary $\partial \Omega$ in an $n$-dimensional Euclidean space $\mathbb{R}^{n}$. Let $\lambda_{i}$ be the $i$-th eigenvalue of the Dirichlet eigenvalue problem of the poly-Laplacian with arbitrary order:

$$
\begin{cases}(-\Delta)^{l} u=\lambda u & \text { in } \Omega, \\ u=\frac{\partial u}{\partial v}=\cdots=\frac{\partial^{l-1} u}{\partial v^{l-1}}=0 & \text { on } \partial \Omega,\end{cases}
$$

where $\Delta$ is the Laplacian in $\mathbb{R}^{n}$ and $v$ denotes the outward unit normal vector field of the boundary $\partial \Omega$. It is well known that the spectrum of this eigenvalue problem is real and discrete:

$$
0<\lambda_{1} \leq \lambda_{2} \leq \lambda_{3} \leq \cdots \rightarrow+\infty
$$

where each $\lambda_{i}$ has finite multiplicity and is repeated according to its multiplicity.

Let $V(\Omega)$ denote the volume of $\Omega$ and let $B_{n}$ denote the volume of the unit ball in $\mathbb{R}^{n}$. When $l=1$, the eigenvalue problem (1-1) is called a fixed membrane problem. In this case, one has Weyl's asymptotic formula

$$
\lambda_{k} \sim \frac{4 \pi^{2}}{\left(B_{n} V(\Omega)\right)^{\frac{2}{n}}} k^{\frac{2}{n}}, \quad k \rightarrow+\infty .
$$

Cheng was partially supported by JSPS Grant-in-Aid for Scientific Research (B) no. 24340013. Qi was partially supported by NSFC grant no. 11171091 . Wei was partially supported by NSFC grant no. 11001087 and the project of Pear River New Star of Guangzhou (grant no. 2012J2200028). Wei is the corresponding author.

MSC2010: 35P15.

Keywords: eigenvalue problem, lower bound for eigenvalues, poly-Laplacian with arbitrary order. 
From the above asymptotic formula, one can obtain

$$
\frac{1}{k} \sum_{i=1}^{k} \lambda_{i} \sim \frac{n}{n+2} \frac{4 \pi^{2}}{\left(B_{n} V(\Omega)\right)^{\frac{2}{n}}} k^{\frac{2}{n}}, \quad k \rightarrow+\infty .
$$

Pólya [1961] proved that

$$
\lambda_{k} \geq \frac{4 \pi^{2}}{\left(B_{n} V(\Omega)\right)^{\frac{2}{n}}} k^{\frac{2}{n}}, \quad \text { for } k=1,2, \ldots,
$$

if $\Omega$ is a tiling domain in $\mathbb{R}^{n}$. Moreover, he proposed the following:

Conjecture of Pólya. If $\Omega$ is a bounded domain in $\mathbb{R}^{n}$, then the $k$-th eigenvalue $\lambda_{k}$ of the fixed membrane problem satisfies

$$
\lambda_{k} \geq \frac{4 \pi^{2}}{\left(B_{n} V(\Omega)\right)^{\frac{2}{n}}} k^{\frac{2}{n}}, \quad \text { for } k=1,2, \ldots
$$

Berezin [1972] and Lieb [1980] gave a partial solution to this conjecture. Li and Yau [1983] proved that

$$
\frac{1}{k} \sum_{i=1}^{k} \lambda_{i} \geq \frac{n}{n+2} \frac{4 \pi^{2}}{\left(B_{n} V(\Omega)\right)^{\frac{2}{n}}} k^{\frac{2}{n}}, \quad \text { for } k=1,2, \ldots
$$

Formula (1-3) shows that (1-6) is sharp in the sense of averages. From (1-6), one can derive

$$
\lambda_{k} \geq \frac{n}{n+2} \frac{4 \pi^{2}}{\left(B_{n} V(\Omega)\right)^{\frac{2}{n}}} k^{\frac{2}{n}}, \quad \text { for } k=1,2, \ldots,
$$

which gives a partial solution for the conjecture of Pólya with a factor $\frac{n}{n+2}$. Melas [2003] has improved the estimate (1-6) to

$$
\frac{1}{k} \sum_{i=1}^{k} \lambda_{i} \geq \frac{n}{n+2} \frac{4 \pi^{2}}{\left(B_{n} V(\Omega)\right)^{\frac{2}{n}}} k^{\frac{2}{n}}+\frac{1}{24(n+2)} \frac{V(\Omega)}{I(\Omega)}, \quad \text { for } k=1,2, \ldots,
$$

where

$$
I(\Omega)=\min _{a \in \mathbb{R}^{n}} \int_{\Omega}|x-a|^{2} d x
$$

is called the moment of inertia of $\Omega$.

When $l=2$, the eigenvalue problem (1-1) is called the clamped plate problem. For the eigenvalues of the clamped plate problem, it follows from [Agmon 1965] and [Pleijel 1950] that

$$
\lambda_{k} \sim \frac{16 \pi^{4}}{\left(B_{n} V(\Omega)\right)^{\frac{4}{n}}} k^{\frac{4}{n}}, \quad k \rightarrow+\infty .
$$


This implies that

$$
\frac{1}{k} \sum_{i=1}^{k} \lambda_{i} \sim \frac{n}{n+4} \frac{16 \pi^{4}}{\left(B_{n} V(\Omega)\right)^{\frac{4}{n}}} k^{\frac{4}{n}}, \quad k \rightarrow+\infty .
$$

Furthermore, Levine and Protter [1985] proved that the eigenvalues of the clamped plate problem satisfy

$$
\frac{1}{k} \sum_{i=1}^{k} \lambda_{i} \geq \frac{n}{n+4} \frac{16 \pi^{4}}{\left(B_{n} V(\Omega)\right)^{\frac{4}{n}}} k^{\frac{4}{n}} .
$$

Formula (1-10) shows that the coefficient of $k^{\frac{4}{n}}$ is the best possible constant. Very recently, Cheng and Wei [2011] obtained the following improvement of (1-11):

$$
\begin{aligned}
& \frac{1}{k} \sum_{i=1}^{k} \lambda_{i} \geq \\
& \quad \frac{n}{n+4} \frac{16 \pi^{4}}{\left(B_{n} V(\Omega)\right)^{\frac{4}{n}}} k^{\frac{4}{n}}+c_{n} \frac{n}{n+2} \frac{4 \pi^{2}}{\left(B_{n} V(\Omega)\right)^{\frac{2}{n}}} \frac{V(\Omega)}{I(\Omega)} k^{\frac{2}{n}}+d_{n}\left(\frac{V(\Omega)}{I(\Omega)}\right)^{2},
\end{aligned}
$$

where $c_{n}$ and $d_{n}$ are constants depending only on the dimension $n$.

When $l \geq 3$, Levine and Protter [1985] proved that

$$
\frac{1}{k} \sum_{i=1}^{k} \lambda_{i} \geq \frac{n}{n+2 l} \frac{(2 \pi)^{2 l}}{\left(B_{n} V(\Omega)\right)^{\frac{2 l}{n}}} k^{\frac{2 l}{n}}, \quad \text { for } k=1,2, \ldots
$$

From the above formula, one can obtain

$$
\lambda_{k} \geq \frac{n}{n+2 l} \frac{(2 \pi)^{2 l}}{\left(B_{n} V(\Omega)\right)^{\frac{2 l}{n}}} k^{\frac{2 l}{n}}, \quad \text { for } k=1,2, \ldots
$$

In this paper we investigate eigenvalues of the Dirichlet eigenvalue problem (1-1) for the Laplacian with any order. We give a significant improvement of (1-13) by adding $l$ lower-order terms than $k^{2 l / n}$ to its right-hand side. In fact, we prove:

Theorem. Let $\Omega$ be a bounded domain in an n-dimensional Euclidean space $\mathbb{R}^{n}$. Let $\lambda_{i}, i=1,2, \ldots$, be the $i$-th eigenvalue of the eigenvalue problem (1-1). Then

$$
\begin{aligned}
\frac{1}{k} \sum_{j=1}^{k} \lambda_{j} \geq & \frac{n}{n+2 l} \frac{(2 \pi)^{2 l}}{\left(B_{n} V(\Omega)\right)^{\frac{2 l}{n}}} k^{\frac{2 l}{n}} \\
& +\frac{n}{n+2 l} \sum_{p=1}^{l} \frac{(l+1-p)}{(24)^{p} n \cdots(n+2 p-2)} \frac{(2 \pi)^{2(l-p)}}{\left(B_{n} V(\Omega)\right)^{\frac{2(l-p)}{n}}}\left(\frac{V(\Omega)}{I(\Omega)}\right)^{p} k^{\frac{2(l-p)}{n}} .
\end{aligned}
$$

Remark. If we take $l=1$, we obtain the inequality (1-8). 


\section{Proof of the Theorem}

Before giving the proof, we introduce some definitions and basic facts about symmetric decreasing rearrangements.

For a bounded domain $\Omega \subset \mathbb{R}^{n}$, the moment of inertia of $\Omega$ is defined by

$$
I(\Omega)=\min _{a \in \mathbb{R}^{n}} \int_{\Omega}|x-a|^{2} d x .
$$

By translating the origin, we may assume that

$$
I(\Omega)=\int_{\Omega}|x|^{2} d x .
$$

Let $\Omega^{*}$ be the symmetric rearrangement of $\Omega$, that is, $\Omega^{*}$ is the open ball centered at the origin with the same volume as $\Omega$. Then

$$
\Omega^{*}=\left\{x \in \mathbb{R}^{n} ;|x|<\left(\frac{V(\Omega)}{B_{n}}\right)^{\frac{1}{n}}\right\} .
$$

By using the symmetric rearrangement $\Omega^{*}$ of $\Omega$, we have

$$
I(\Omega)=\int_{\Omega}|x|^{2} d x \geq \int_{\Omega^{*}}|x|^{2} d x=\frac{n}{n+2} V(\Omega)\left(\frac{V(\Omega)}{B_{n}}\right)^{\frac{2}{n}} .
$$

Let $f$ be a nonnegative continuous function on $\Omega$. We consider its distribution function $\mu_{f}(t)$ defined by

$$
\mu_{f}(t)=\operatorname{Vol}(\{x \in \Omega ; f(x)>t\}) .
$$

The distribution function can be viewed as a function from $[0,+\infty)$ to $[0, V(\Omega)]$. The symmetric decreasing rearrangement $f^{*}$ of $f$ is defined by

$$
f^{*}(x)=\inf \left\{t \geq 0 ; \mu_{f}(t)<B_{n}|x|^{n}\right\}, \quad \text { for } x \in \Omega^{*} .
$$

By definition, we know that $f^{*}(x)$ is a radially symmetric function and

$$
\operatorname{Vol}(\{x \in \Omega ; f(x)>t\})=\operatorname{Vol}\left(\left\{x \in \Omega^{*} ; f^{*}(x)>t\right\}\right) \text { for all } t>0 .
$$

Let $f^{*}(x)=\phi(|x|)$. Then one gets that $\phi:[0,+\infty) \rightarrow[0, \sup f]$ is a decreasing function of $|x|$. We may assume that $\phi$ is absolutely continuous. It is well known that

$$
\int_{\Omega} f(x) d x=\int_{\Omega^{*}} f^{*}(x) d x=n B_{n} \int_{0}^{+\infty} s^{n-1} \phi(s) d s
$$

and

$$
\int_{\Omega}|x|^{2 l} f(x) d x \geq \int_{\Omega^{*}}|x|^{2 l} f^{*}(x) d x=n B_{n} \int_{0}^{+\infty} s^{n+2 l-1} \phi(s) d s .
$$


Good sources of further information on rearrangements are [Bandle 1980; Pólya and Szegó 1951].

One gets from the coarea formula that

$$
\mu_{f}(t)=\int_{t}^{\sup f} \int_{\{f=s\}}|\nabla f|^{-1} d \sigma_{s} d s .
$$

Since $f^{*}$ is radial, we have

$$
\begin{aligned}
\mu_{f}(\phi(s)) & =\operatorname{Vol}\{x \in \Omega ; f(x)>\phi(s)\}=\operatorname{Vol}\left\{x \in \Omega^{*} ; f^{*}(x)>\phi(s)\right\} \\
& =\operatorname{Vol}\left\{x \in \Omega^{*} ; \phi(|x|)>\phi(s)\right\}=B_{n} s^{n} .
\end{aligned}
$$

It follows that

$$
n B_{n} s^{n-1}=\mu_{f}^{\prime}(\phi(s)) \phi^{\prime}(s)
$$

for almost every $s$. Putting $\tau:=\sup |\nabla f|$, we obtain from the above equations and the isoperimetric inequality that

$-\mu_{f}^{\prime}(\phi(s))=\int_{\{f=\phi(s)\}}|\nabla f|^{-1} d \sigma_{\phi(s)} \geq \tau^{-1} \operatorname{Vol}_{n-1}(\{f=\phi(s)\}) \geq \tau^{-1} n B_{n} s^{n-1}$.

Therefore, one obtains, for almost every $s$,

$$
-\tau \leq \phi^{\prime}(s) \leq 0 .
$$

In order to prove our theorem, we need the following lemma.

Lemma. Let $b \geq 1$ and $\eta, A>0$, and let $\psi:[0,+\infty) \rightarrow[0,+\infty)$ be a decreasing, absolutely continuous function such that

$$
-\eta \leq \psi^{\prime}(s) \leq 0, \quad A=\int_{0}^{+\infty} s^{b-1} \psi(s) d s .
$$

For any positive integer l, let

$$
A_{l}:=\int_{0}^{+\infty} s^{b+2 l-1} \psi(s) d s .
$$

Then, we have

$$
A_{l} \geq \frac{1}{b+2 l}\left[(b A)^{\frac{b+2 l}{b}} \psi(0)^{-\frac{2 l}{b}}+\sum_{p=1}^{l} \frac{(l+1-p)(b A)^{\frac{b+2(l-p)}{b}} \psi(0)^{\frac{2 p b-2(l-p)}{b}}}{6^{p} b \cdots(b+2 p-2) \eta^{2 p}}\right] .
$$

Proof. The proof is by induction. Firstly, one can get from the lemma of [Melas 2003] that

$$
A_{1}=\int_{0}^{+\infty} s^{b+1} \psi(s) d s \geq \frac{1}{b+2}\left[(b A)^{\frac{b+2}{b}} \psi(0)^{-\frac{2}{b}}+\frac{A \psi(0)^{2}}{6 \eta^{2}}\right] .
$$


To prove the induction step, we assume the statement holds for $l=r$, that is, $A_{r} \geq \frac{1}{b+2 r}\left[(b A)^{\frac{b+2 r}{b}} \psi(0)^{-\frac{2 r}{b}}+\sum_{p=1}^{r} \frac{(r+1-p)(b A)^{\frac{b+2(r-p)}{b}} \psi(0)^{\frac{2 p b-2(r-p)}{b}}}{6^{p} b \cdots(b+2 p-2) \eta^{2 p}}\right]$.

Since the formula (2-5) holds for any $b \geq 1$, we have

$$
\begin{aligned}
& A_{r+1} \\
& =\int_{0}^{+\infty} s^{b+2 r+1} \psi(s) d s \\
& \geq \frac{1}{b+2 r+2}\left\{\left[(b+2 r) A_{r}\right]^{\frac{b+2 r+2}{b+2 r}} \psi(0)^{-\frac{2}{b+2 r}}+\frac{A_{r} \psi(0)^{2}}{6 \eta^{2}}\right\} \\
& \geq \frac{\psi(0)^{-\frac{2}{b+2 r}}}{b+2 r+2} \\
& \times\left[(b A)^{\frac{b+2 r}{b}} \psi(0)^{-\frac{2 r}{b}}+\sum_{p=1}^{r} \frac{(r+1-p)(b A)^{\frac{b+2(r-p)}{b}} \psi(0)^{\frac{2 p b-2(r-p)}{b}}}{6^{p} b \cdots(b+2 p-2) \eta^{2 p}}\right]^{\frac{b+2 r+2}{b+2 r}} \\
& +\frac{1}{(b+2 r)(b+2 r+2)} \sum_{p=1}^{r} \frac{(r+1-p)(b A)^{\frac{b+2(r-p)}{b}} \psi(0)^{\frac{2(p+1) b-2(r-p)}{b}}}{6^{p+1} b \cdots(b+2 p-2) \eta^{2 p+2}} \\
& +\frac{(b A)^{\frac{b+2 r}{b}} \psi(0)^{\frac{2 b-2 r}{b}}}{6(b+2 r)(b+2 r+2) \eta^{2}} \\
& =\frac{\psi(0)^{-\frac{2}{b+2 r}}}{b+2 r+2}\left[(b A) p^{\frac{b+2 r}{b}} \psi(0)^{-\frac{2 r}{b}}\right]^{\frac{b+2 r+2}{b+2 r}} \\
& \times\left[1+\sum_{p=1}^{r} \frac{(r+1-p)(b A)^{\frac{-2 p}{b}} \psi(0)^{\frac{2 p b+2 p}{b}}}{6^{p} b \cdots(b+2 p-2) \eta^{2 p}}\right]^{\frac{b+2 r+2}{b+2 r}}+\frac{(b A)^{\frac{b+2 r}{b}} \psi(0)^{\frac{2 b-2 r}{b}}}{6(b+2 r)(b+2 r+2) \eta^{2}} \\
& +\frac{1}{(b+2 r)(b+2 r+2)} \sum_{p=2}^{r+1} \frac{(r+2-p)(b A)^{\frac{b+2 r-2 p+2}{b}} \psi(0)^{\frac{2 p b-2 r+2 p-2}{b}}}{6^{p} b \cdots(b+2 p-4) \eta^{2 p}} \\
& =\frac{(b A)^{\frac{b+2 r+2}{b}} \psi(0)^{-\frac{2 r+2}{b}}}{b+2 r+2}\left[1+\sum_{p=1}^{r} \frac{(r+1-p)(b A)^{\frac{-2 p}{b}} \psi(0)^{\frac{2 p b+2 p}{b}}}{6^{p} b \cdots(b+2 p-2) \eta^{2 p}}\right]^{\frac{b+2 r+2}{b+2 r}} \\
& +\frac{(b A)^{\frac{b+2 r}{b}} \psi(0)^{\frac{2 b-2 r}{b}}}{6(b+2 r)(b+2 r+2) \eta^{2}}+\frac{(b A) \psi(0)^{2(r+1)}}{6^{r+1} b \cdots(b+2 r+2) \eta^{2(r+1)}} \\
& +\frac{1}{(b+2 r)(b+2 r+2)} \sum_{p=2}^{r} \frac{(r+2-p)(b A)^{\frac{b+2(r+1-p)}{b}} \psi(0)^{\frac{2 p b-2(r+1-p)}{b}}}{6^{p} b \cdots(b+2 p-4) \eta^{2 p}} \text {. }
\end{aligned}
$$


It follows from the Taylor formula that

$$
\begin{aligned}
& A_{r+1} \\
& \geq \frac{1}{b+2 r+2}(b A)^{\frac{b+2 r+2}{b}} \psi(0)^{-\frac{2 r+2}{b}} \\
& \times\left[1+\frac{b+2 r+2}{b+2 r} \sum_{p=1}^{r} \frac{(r+1-p)(b A)^{\frac{-2 p}{b}} \psi(0)^{\frac{2 p b+2 p}{b}}}{6^{p} b \cdots(b+2 p-2) \eta^{2 p}}\right] \\
& +\frac{(b A)^{\frac{b+2 r}{b}} \psi(0)^{\frac{2 b-2 r}{b}}}{6(b+2 r)(b+2 r+2) \eta^{2}}+\frac{(b A) \psi(0)^{2(r+1)}}{6^{r+1} b \cdots(b+2 r+2) \eta^{2(r+1)}} \\
& +\frac{1}{(b+2 r)(b+2 r+2)} \sum_{p=2}^{r} \frac{(r+2-p)(b A)^{\frac{b+2(r+1-p)}{b}} \psi(0)^{\frac{2 p b-2(r+1-p)}{b}}}{6^{p} b \cdots(b+2 p-4) \eta^{2 p}} \\
& =\frac{1}{b+2 r+2}(b A)^{\frac{b+2 r+2}{b}} \psi(0)^{-\frac{2 r+2}{b}} \\
& +\frac{1}{b+2 r} \sum_{p=1}^{r} \frac{(r+1-p)(b A)^{\frac{b+2(r+1-p)}{b}} \psi(0)^{\frac{2 p b-2(r+1-p)}{b}}}{6^{p} b \cdots(b+2 p-2) \eta^{2 p}} \\
& +\frac{(b A)^{\frac{b+2 r}{b}} \psi(0)^{\frac{2 b-2 r}{b}}}{6(b+2 r)(b+2 r+2) \eta^{2}}+\frac{(b A) \psi(0)^{2(r+1)}}{6^{r+1} b \cdots(b+2 r+2) \eta^{2(r+1)}} \\
& +\frac{1}{(b+2 r)(b+2 r+2)} \sum_{p=2}^{r} \frac{(r+2-p)(b A)^{\frac{b+2(r+1-p)}{b}} \psi(0)^{\frac{2 p b-2(r+1-p)}{b}}}{6^{p} b \cdots(b+2 p-4) \eta^{2 p}} \\
& =\frac{1}{b+2 r+2}(b A)^{\frac{b+2 r+2}{b}} \psi(0)^{-\frac{2 r+2}{b}} \\
& +\left[\frac{r}{b(b+2 r)}+\frac{1}{(b+2 r)(b+2 r+2)}\right] \frac{1}{6 \eta^{2}}(b A)^{\frac{b+2 r}{b}} \psi(0)^{\frac{2 b-2 r}{b}} \\
& +\sum_{p=2}^{r}\left[\frac{r+1-p}{b+2 r}+\frac{(r+2-p)(b+2 p-2)}{(b+2 r)(b+2 r+2)}\right] \frac{(b A)^{\frac{b+2(r+1-p)}{b}} \psi(0)^{\frac{2 p b-2(r+1-p)}{b}}}{6^{p} b \cdots(b+2 p-2) \eta^{2 p}} \\
& (b A) \psi(0)^{2(r+1)} \\
& +\frac{(b+2 r+2) \eta^{2(r+1)}}{6^{r+1} b \cdots(b+2)} \\
& \geq \frac{1}{b+2(r+1)}(b A)^{\frac{b+2(r+1)}{b}} \psi(0)^{-\frac{2(r+1)}{b}} \\
& +\frac{1}{b+2(r+1)} \sum_{p=1}^{r+1} \frac{(r+2-p)(b A)^{\frac{b+2(r+1-p)}{b}} \psi(0)^{\frac{2 p b-2(r+1-p)}{b}}}{6^{p} b \cdots(b+2 p-2) \eta^{2 p}} .
\end{aligned}
$$


This completes the proof of the lemma.

Proof of the Theorem. Let $u_{j}$ be an orthonormal eigenfunction corresponding to the eigenvalue $\lambda_{j}$, that is, $u_{j}$ satisfies

$$
\begin{cases}(-\Delta)^{l} u_{j}=\lambda_{j} u_{j}, & \text { in } \Omega, \\ u_{j}=\frac{\partial u_{j}}{\partial v}=\cdots=\frac{\partial^{l-1} u_{j}}{\partial v^{l-1}}=0, & \text { on } \partial \Omega, \\ \int_{\Omega} u_{i} u_{j}=\delta_{i j}, & \text { for any } i, j .\end{cases}
$$

Thus, $\left\{u_{j}\right\}_{j=1}^{\infty}$ forms an orthonormal basis of $L^{2}(\Omega)$. We define a function $\varphi_{j}$ by

$$
\varphi_{j}(x)= \begin{cases}u_{j}(x), & x \in \Omega, \\ 0, & x \in \mathbb{R}^{n} \backslash \Omega .\end{cases}
$$

The Fourier transform $\widehat{\varphi}_{j}(z)$ of $\varphi_{j}(x)$ is then given by

$$
\widehat{\varphi}_{j}(z)=(2 \pi)^{-n / 2} \int_{\mathbb{R}^{n}} \varphi_{j}(x) e^{i\langle x, z\rangle} d x=(2 \pi)^{-n / 2} \int_{\Omega} u_{j}(x) e^{i\langle x, z\rangle} d x .
$$

We fix $k \geq 1$ and set

$$
f(z)=\sum_{j=1}^{k}\left|\widehat{\varphi}_{j}(z)\right|^{2}, \quad \text { for } z \in \mathbb{R}^{n} .
$$

From Bessel's inequality, it follows that

$$
\begin{aligned}
0 \leq f(z) & =\sum_{j=1}^{k}\left|\widehat{\varphi}_{j}(z)\right|^{2}=(2 \pi)^{-n} \sum_{j=1}^{k}\left|\int_{\Omega} u_{j}(x) e^{i\langle x, z\rangle} d x\right|^{2} \\
& \leq(2 \pi)^{-n} \int_{\Omega}\left|e^{i\langle x, z\rangle}\right|^{2} d x=(2 \pi)^{-n} V(\Omega) .
\end{aligned}
$$

By Parseval's identity, we have

$$
\begin{aligned}
\int_{\mathbb{R}^{n}} f(z) d z & =\sum_{j=1}^{k} \int_{\mathbb{R}^{n}}\left|\widehat{\varphi}_{j}(z)\right|^{2} d z=\sum_{j=1}^{k} \int_{\mathbb{R}^{n}} \varphi_{j}^{2}(x) d x \\
& =\sum_{j=1}^{k} \int_{\Omega} u_{j}^{2}(x) d x=k .
\end{aligned}
$$

Furthermore, we deduce from integration by parts and Parseval's identity that 
(2-11) $\int_{\mathbb{R}^{n}}|z|^{2 l} f(z) d z$

$$
\begin{aligned}
= & \sum_{j=1}^{k} \int_{\mathbb{R}^{n}}|z|^{2 l}\left|\widehat{\varphi}_{j}(z)\right|^{2} d z \\
= & \sum_{j=1}^{k} \int_{\mathbb{R}^{n}}|z|^{2 l}\left|(2 \pi)^{-n / 2} \int_{\Omega} u_{j}(x) e^{i\langle x, z\rangle} d x\right|^{2} d z \\
= & \sum_{j=1}^{k} \sum_{r_{1}, \ldots, r_{l}=1}^{n} \int_{\mathbb{R}^{n}}\left|(2 \pi)^{-n / 2} \int_{\Omega} z_{r_{1}} \cdots z_{r_{l}} u_{j}(x) e^{i\langle x, z\rangle} d x\right|^{2} d z \\
= & \sum_{j=1}^{k} \sum_{r_{1}, \ldots, r_{l}=1}^{n} \int_{\mathbb{R}^{n}}\left|(2 \pi)^{-n / 2} \int_{\Omega} u_{j}(x) \frac{\partial^{l} e^{i\langle x, z\rangle}}{\partial x_{r_{1}} \cdots \partial x_{r_{l}}} d x\right|^{2} d z \\
= & \sum_{j=1}^{k} \sum_{r_{1}, \ldots, r_{l}=1}^{n} \int_{\mathbb{R}^{n}}\left|(2 \pi)^{-n / 2} \int_{\Omega} \frac{\partial^{l} u_{j}(x)}{\partial x_{r_{1}} \cdots \partial x_{r_{l}}} e^{i\langle x, z\rangle} d x\right|^{2} d z \\
= & \sum_{j=1}^{k} \sum_{r_{1}, \ldots, r_{l}=1}^{n} \int_{\mathbb{R}^{n}}\left|\frac{\partial^{l} u_{j}}{\partial x_{r_{1}} \cdots \partial x_{r_{l}}}\right|^{2} d z \\
= & \sum_{j=1}^{k} \sum_{k}^{n} \int_{\mathbb{R}_{1}, \ldots, r_{l}=1}\left(\frac{\partial^{l} u_{j}}{\partial x_{r_{1}} \cdots \partial x_{r_{l}}}\right)^{2} d x \\
= & \sum_{j=1}^{k} \int_{\Omega} u_{j}(-\Delta)^{l} u_{j} d x=\sum_{j=1}^{k} \lambda_{j} . \\
& =1 \\
&
\end{aligned}
$$

Since

$$
\nabla \widehat{\varphi}_{j}(z)=(2 \pi)^{-n / 2} \int_{\Omega} i x u_{j}(x) e^{i\langle x, z\rangle} d x,
$$

we obtain from Bessel's inequality that

$$
\sum_{j=1}^{k}\left|\nabla \widehat{\varphi}_{j}(z)\right|^{2} \leq(2 \pi)^{-n} \int_{\Omega}\left|i x e^{i\langle x, z\rangle}\right|^{2} d x=(2 \pi)^{-n} I(\Omega) .
$$

It follows from (2-9), (2-13) and the Cauchy-Schwarz inequality that, for every $z \in \mathbb{R}^{n}$,

$$
\begin{aligned}
|\nabla f(z)| & \leq 2\left(\sum_{j=1}^{k}\left|\widehat{\varphi}_{j}(z)\right|^{2}\right)^{1 / 2}\left(\sum_{j=1}^{k}\left|\nabla \widehat{\varphi}_{j}(z)\right|^{2}\right)^{1 / 2} \\
& \leq 2(2 \pi)^{-n} \sqrt{V(\Omega) I(\Omega)}
\end{aligned}
$$


Using the symmetric decreasing rearrangement $f^{*}$ of $f$ and noting that

$$
f^{*}(x)=\phi(|x|), \quad \tau=\sup |\nabla f| \leq 2(2 \pi)^{-n} \sqrt{V(\Omega) I(\Omega)}:=\eta,
$$

we obtain, from (2-4),

$$
-\eta \leq-\tau \leq \phi^{\prime}(s) \leq 0
$$

for almost every $s$. According to (2-2) and (2-10), we infer

$$
k=\int_{\mathbb{R}^{n}} f(z) d z=\int_{\mathbb{R}^{n}} f^{*}(z) d z=n B_{n} \int_{0}^{+\infty} s^{n-1} \phi(s) d s .
$$

From (2-3) and (2-11), we obtain

$$
\begin{aligned}
\sum_{j=1}^{k} \lambda_{j} & =\int_{\mathbb{R}^{n}}|z|^{2 l} f(z) d z \geq \int_{\mathbb{R}^{n}}|z|^{2 l} f^{*}(z) d z \\
& =n B_{n} \int_{0}^{+\infty} s^{n+2 l-1} \phi(s) d s .
\end{aligned}
$$

Now, we can apply the Lemma to the function $\phi$ with

$$
b=n, \quad A=\frac{k}{n B_{n}}, \quad \eta=2(2 \pi)^{-n} \sqrt{V(\Omega) I(\Omega)} .
$$

We conclude that

$$
\begin{aligned}
\sum_{j=1}^{k} \lambda_{j} \geq & \frac{n B_{n}}{n+2 l}\left(\frac{k}{B_{n}}\right)^{\frac{n+2 l}{n}} \phi(0)^{-\frac{2 l}{n}} \\
& +\frac{n B_{n}}{n+2 l} \sum_{p=1}^{l} \frac{(l+1-p)}{6^{p} n \cdots(n+2 p-2) \eta^{2 p}}\left(\frac{k}{B_{n}}\right)^{\frac{n+2 l-2 p}{n}} \phi(0)^{\frac{2 p n+2 p-2 l}{n}}
\end{aligned}
$$

Note that $0<\phi(0) \leq \sup f \leq(2 \pi)^{-n} V(\Omega)$. Hence we consider the function $F$ defined by

$$
\begin{aligned}
(2-20) \quad F(t)= & \frac{n B_{n}}{n+2 l}\left(\frac{k}{B_{n}}\right)^{\frac{n+2 l}{n}} t^{-\frac{2 l}{n}} \\
& +\frac{n B_{n}}{n+2 l} \sum_{p=1}^{l} \frac{(l+1-p)}{6^{p} n \cdots(n+2 p-2) \eta^{2 p}}\left(\frac{k}{B_{n}}\right)^{\frac{n+2 l-2 p}{n}} t^{\frac{2 p n+2 p-2 l}{n}},
\end{aligned}
$$

for $t \in\left(0,(2 \pi)^{-n} V(\Omega)\right]$. From (2-1), we have

$$
\eta \geq(2 \pi)^{-n} B_{n}^{-\frac{1}{n}} V(\Omega)^{\frac{n+1}{n}} .
$$


By a direct calculation, one gets from $B_{n}=\frac{2 \pi^{n / 2}}{n \Gamma(n / 2)}$ that

$$
\frac{B_{n}^{4 / n}}{(2 \pi)^{2}}<\frac{1}{2},
$$

where $\Gamma\left(\frac{n}{2}\right)$ is the gamma function. Thus, it follows from (2-21) and (2-22) that

$$
\begin{aligned}
& F^{\prime}(t)=\frac{2 B_{n} t^{-\frac{n+2 l}{n}}}{n+2 l}\left(\frac{k}{B_{n}}\right)^{\frac{n+2 l}{n}}\left[-l+\sum_{p=1}^{l} \frac{(l+1-p)(p n+p-l) t^{\frac{2 p(n+1)}{n}}}{6^{p} n \cdots(n+2 p-2) \eta^{2 p}}\left(\frac{k}{B_{n}}\right)^{-\frac{2 p}{n}}\right] \\
& \leq \frac{2 B_{n}}{n+2 l}\left(\frac{k}{B_{n}}\right)^{\frac{n+2 l}{n}} t^{-\frac{n+2 l}{n}}\left[-l+\sum_{p>\frac{l}{n+1}}^{l} \frac{(l+1-p)(p n+p-l)}{6^{p} n \cdots(n+2 p-2)}\left(\frac{B_{n}^{\frac{4}{n}}}{(2 \pi)^{2}}\right)^{p}\right] \\
& <\frac{2 B_{n}}{n+2 l}\left(\frac{k}{B_{n}}\right)^{\frac{n+2 l}{n}} t^{-\frac{n+2 l}{n}}\left[-l+\sum_{p>\frac{l}{n+1}}^{l} \frac{(l+1-p)(p n+p-l)}{(12)^{p} n \cdots(n+2 p-2)}\right] \\
& <\frac{2 B_{n}}{n+2 l}\left(\frac{k}{B_{n}}\right)^{\frac{n+2 l}{n}} t^{-\frac{n+2 l}{n}}\left[-l+\frac{l(n+1-l)}{12 n}+\sum_{\substack{p>\frac{l}{n+1} \\
p \neq 1}}^{l} \frac{p^{2} n(n+1)}{(12)^{p} n \cdots(n+2 p-2)}\right] \\
& <\frac{2 B_{n}}{n+2 l}\left(\frac{k}{B_{n}}\right)^{\frac{n+2 l}{n}} t^{-\frac{n+2 l}{n}}\left[-l+\frac{l}{12}+\sum_{\substack{p>\frac{l}{n+1} \\
p \neq 1}}^{l} \frac{p^{2}}{(12)^{p}}\right] \\
& <\frac{2 B_{n}}{n+2 l}\left(\frac{k}{B_{n}}\right)^{\frac{n+2 l}{n}} t^{-\frac{n+2 l}{n}}\left[-l+\frac{l}{12}+\frac{1}{12}\right]<0 .
\end{aligned}
$$

We obtain that $F(t)$ is a decreasing function on $\left(0,(2 \pi)^{-n} V(\Omega)\right]$. Then we can replace $\phi(0)$ by $(2 \pi)^{-n} V(\Omega)$ in (2-19), namely,

$$
\begin{aligned}
\sum_{j=1}^{k} \lambda_{j} \geq & \frac{n}{n+2 l} \frac{(2 \pi)^{2 l}}{\left(B_{n} V(\Omega)\right)^{\frac{2 l}{n}}} k^{\frac{n+2 l}{n}} \\
& +\frac{n}{n+2 l} \sum_{p=1}^{l} \frac{(l+1-p)}{6^{p} n \cdots(n+2 p-2) \eta^{2 p}} \frac{(V(\Omega))^{\frac{2 p n+2 p-2 l}{n}}}{(2 \pi)^{2 p n+2 p-2 n} B_{n}^{\frac{2 l-2 p}{n}}} k^{\frac{n+2 l-2 p}{n}} \\
= & \frac{n}{n+2 l} \frac{(2 \pi)^{2 l}}{\left(B_{n} V(\Omega)\right)^{\frac{2 l}{n}}} k^{\frac{n+2 l}{n}} \\
& +\frac{n}{n+2 l} \sum_{p=1}^{l} \frac{(l+1-p)}{24^{p} n \cdots(n+2 p-2)} \frac{(2 \pi)^{2(l-p)}}{\left(B_{n} V(\Omega)\right)^{\frac{2(l-p)}{n}}}\left(\frac{V(\Omega)}{I(\Omega)}\right)^{p} k^{\frac{n+2(l-p)}{n}} .
\end{aligned}
$$

This completes the proof of the Theorem. 


\section{References}

[Agmon 1965] S. Agmon, "On kernels, eigenvalues, and eigenfunctions of operators related to elliptic problems", Comm. Pure Appl. Math. 18 (1965), 627-663. MR 33 \#6446 Zbl 0151.20203

[Bandle 1980] C. Bandle, Isoperimetric inequalities and applications, Monographs and Studies in Mathematics 7, Pitman, Boston, 1980. MR 81e:35095 Zbl 0436.35063

[Berezin 1972] F. A. Berezin, "Ковариантные и контравариантные символы операторов", Izv. Akad. Nauk SSSR Ser. Mat. 36:5 (1972), 1134-1167. Translated as "Covariant and contravariant symbols of operators" in Math. USSR-Izv. 6:5 (1972), 1117-1151. MR 50 \#2996 Zbl 0259.47004

[Cheng and Wei 2011] Q.-M. Cheng and G. Wei, "A lower bound for eigenvalues of a clamped plate problem”, Calc. Var. Partial Differential Equations 42:3-4 (2011), 579-590. MR 2012i:35070 Zbl 1234.35158

[Levine and Protter 1985] H. A. Levine and M. H. Protter, "Unrestricted lower bounds for eigenvalues for classes of elliptic equations and systems of equations with applications to problems in elasticity", Math. Methods Appl. Sci. 7:2 (1985), 210-222. MR 87d:35101 Zbl 0591.35050

[Li and Yau 1983] P. Li and S. T. Yau, "On the Schrödinger equation and the eigenvalue problem", Comm. Math. Phys. 88:3 (1983), 309-318. MR 84k:58225 Zbl 0554.35029

[Lieb 1980] E. H. Lieb, "The number of bound states of one-body Schrödinger operators and the Weyl problem", pp. 241-252 in Geometry of the Laplace operator (Honolulu, HI, 1979), edited by R. Osserman and A. Weinstein, Proc. Sympos. Pure Math. 36, American Mathematical Society, Providence, RI, 1980. Also in The stability of matter: from atoms to stars, selecta of Elliott $\mathrm{H}$. Lieb, 4th ed., edited by Thirring, W., 245-256, Springer, Berlin, 2005. MR 82i:35134 Zbl 0445.58029

[Melas 2003] A. D. Melas, "A lower bound for sums of eigenvalues of the Laplacian", Proc. Amer. Math. Soc. 131:2 (2003), 631-636. MR 2003i:35218 Zbl 1015.58011

[Pleijel 1950] Å. Pleijel, "On the eigenvalues and eigenfunctions of elastic plates", Comm. Pure Appl. Math. 3 (1950), 1-10. MR 12,265a Zbl 0040.05403

[Pólya 1961] G. Pólya, "On the eigenvalues of vibrating membranes”, Proc. London Math. Soc. (3) 11 (1961), 419-433. MR 23 \#B2256 Zbl 0107.41805

[Pólya and Szegó 1951] G. Pólya and G. Szegö, Isoperimetric inequalities in mathematical physics, Annals of Mathematics Studies 27, Princeton University Press, Princeton, NJ, 1951. MR 13,270d Zbl 0044.38301 
Received December 14, 2010.

QING-MING CHENG

Department of ApPlied Mathematics

FACULTY OF SCIENCES

FUKUOKA UNIVERSITY

FUKUOKA 814-0180

JAPAN

cheng@fukuoka-u.ac.jp

XUERONG QI

DEPARTMENT OF MATHEMATICS

ZHENGZHOU UNIVERSITY

450001 ZHENGZHOU

CHINA

xrqi@zzu.edu.cn

GUOXIN WEI

SCHOOL OF Mathematical SCIENCES

SOUTH CHINA NORMAL UNIVERSITY

510631 GUANGZHOU

CHINA

weiguoxin@tsinghua.org.cn

weigx@scnu.edu.cn 


\title{
PACIFIC JOURNAL OF MATHEMATICS
}

\author{
msp.org/pjm
}

Founded in 1951 by E. F. Beckenbach (1906-1982) and F. Wolf (1904-1989)

\section{EDITORS}

V. S. Varadarajan (Managing Editor)

Department of Mathematics

University of California

Los Angeles, CA 90095-1555

pacific@math.ucla.edu

Paul Balmer

Department of Mathematics

University of California

Los Angeles, CA 90095-1555

balmer@math.ucla.edu

Daryl Cooper

Department of Mathematics

University of California

Santa Barbara, CA 93106-3080 cooper@math.ucsb.edu

Jiang-Hua $\mathrm{Lu}$

Department of Mathematics

The University of Hong Kong

Pokfulam Rd., Hong Kong jhlu@maths.hku.hk
Don Blasius

Department of Mathematics University of California

Los Angeles, CA 90095-1555

blasius@math.ucla.edu

Robert Finn

Department of Mathematics Stanford University

Stanford, CA 94305-2125

finn@math.stanford.edu

Sorin Popa

Department of Mathematics

University of California

Los Angeles, CA 90095-1555

popa@math.ucla.edu

Paul Yang

Department of Mathematics

Princeton University

Princeton NJ 08544-1000

yang@math.princeton.edu

\section{PRODUCTION}

Silvio Levy, Scientific Editor, production@msp.org

\section{SUPPORTING INSTITUTIONS}

ACADEMIA SINICA, TAIPEI

CALIFORNIA INST. OF TECHNOLOGY

INST. DE MATEMÁTICA PURA E APLICADA

KEIO UNIVERSITY

MATH. SCIENCES RESEARCH INSTITUTE

NEW MEXICO STATE UNIV.

OREGON STATE UNIV.

\author{
STANFORD UNIVERSITY \\ UNIV. OF BRITISH COLUMBIA \\ UNIV. OF CALIFORNIA, BERKELEY \\ UNIV. OF CALIFORNIA, DAVIS \\ UNIV. OF CALIFORNIA, LOS ANGELES \\ UNIV. OF CALIFORNIA, RIVERSIDE \\ UNIV. OF CALIFORNIA, SAN DIEGO \\ UNIV. OF CALIF., SANTA BARBARA
}

\author{
Vyjayanthi Chari \\ Department of Mathematics \\ University of California \\ Riverside, CA 92521-0135 \\ chari@math.ucr.edu \\ Kefeng Liu \\ Department of Mathematics \\ University of California \\ Los Angeles, CA 90095-1555 \\ liu@math.ucla.edu \\ Jie Qing \\ Department of Mathematics \\ University of California \\ Santa Cruz, CA 95064 \\ qing@cats.ucsc.edu
}

These supporting institutions contribute to the cost of publication of this Journal, but they are not owners or publishers and have no responsibility for its contents or policies.

See inside back cover or msp.org/pjm for submission instructions.

The subscription price for 2013 is US \$400/year for the electronic version, and \$485/year for print and electronic.

Subscriptions, requests for back issues and changes of subscribers address should be sent to Pacific Journal of Mathematics, P.O. Box 4163, Berkeley, CA 94704-0163, U.S.A. The Pacific Journal of Mathematics is indexed by Mathematical Reviews, Zentralblatt MATH, PASCAL CNRS Index, Referativnyi Zhurnal, Current Mathematical Publications and the Science Citation Index.

The Pacific Journal of Mathematics (ISSN 0030-8730) at the University of California, c/o Department of Mathematics, 798 Evans Hall \#3840, Berkeley, CA 94720-3840, is published monthly except July and August. Periodical rate postage paid at Berkeley, CA 94704, and additional mailing offices. POSTMASTER: send address changes to Pacific Journal of Mathematics, P.O. Box 4163, Berkeley, CA 94704-0163.

PJM peer review and production are managed by EditFLOW ${ }^{\circledR}$ from Mathematical Sciences Publishers.

PUBLISHED BY

mathematical sciences publishers

nonprofit scientific publishing

http://msp.org/

(C) 2013 Mathematical Sciences Publishers 


\section{PACIFIC JOURNAL OF MATHEMATICS}

Volume $262 \quad$ No. $1 \quad$ March 2013

On the second $K$-group of a rational function field

KARIM JOHANNES BECHER and MÉLANIE RACZEK

On existence of a classical solution to a generalized Kelvin-Voigt model

Miroslav BulíčEK, Petr KaPliCKÝ and Mark

STEINHAUER

A lower bound for eigenvalues of the poly-Laplacian with arbitrary order

Qing-Ming Cheng, Xuerong Qi and GuOXIN Wei

Quiver algebras, path coalgebras and coreflexivity

SORIN DĂSCĂLESCU, MIODRAG C. IOVANOV and CONSTANTIN

NĂSTĂSESCU

A positive density of fundamental discriminants with large regulator

ÉTIENNE FOUVRY and FLORENT JOUVE

On the isentropic compressible Euler equation with adiabatic index $\gamma=1$

Dong Li, ChANGXing MiaO and XiaOyi Zhang

Symmetric regularization, reduction and blow-up of the planar three-body problem

RICHARD MOECKEL and RICHARD MONTGOMERY

Canonical classes and the geography of nonminimal Lefschetz fibrations over $S^{2}$

YOSHIHISA SATO

Hilbert-Kunz invariants and Euler characteristic polynomials 